\title{
Implicative Algebras
}

\section{Venkateswarlu Kolluru* and Berhanu Bekele**}

Department of Mathematics, Faculty of Computer and Mathematical Sciences, Addis Ababa University, Addis Ababa, Ethiopia(*drkvenkateswarlu@gmail.com,**berhanufk@yahoo.co.uk)

\begin{abstract}
In this paper we introduce the concept of implicative algebras which is an equivalent definition of lattice implication algebra of $\mathrm{Xu}$ (1993) and further we prove that it is a regular Autometrized Algebra. Further we remark that the binary operation $\rightarrow$ on lattice implicative algebra can never be associative.
\end{abstract}

Key words: Implicative algebra, lattice implication algebras, Autometrized Algebras, regular Autometrized Algebras

Mathematics subject classification 2000: Primary 3G 10, 06A06, 06A11

\section{INTRODUCTION}

The concept of lattice implication algebras is due to $\mathrm{Xu}$ (1993). In his paper, he introduced the concept of lattice implication algebra and quasi lattice implication algebra as a bounded lattice satisfying a system of axioms (see definition [2] below) and studied certain properties. Later many authors like Jun et al. (1998) have studied the properties of filters and fuzzy filters of lattice implication algebras and quasi lattice implication algebras. Also Zhu Yiquan and Tu (2001) have introduced an equivalent definition for lattice implication algebra in [4]. In this paper, we also give an equivalent definition of implication lattices of Xu (1993) by simplifying the axioms of his definition and call implicative algebras (see definition1.1). For instance, $\mathrm{Xu}$ (1993) proposed lattice implicative algebras as a bounded lattice with involution but in the sense of our definition even lattice structure is not required .Using our definition (implicative algebras) we obtain lattice implication algebra of Xu (1993).

This paper is divided into two sections. In the first section we introduce the concept of implicative algebras and obtain certain properties. Further we prove that implicative algebra is equipped with a structure of a bounded lattice and prove that it is a lattice implication algebra (see theorem 1.13). It is also observed that " $\rightarrow$ " can never be associative. (see remark 1.14). In section 2, we introduce two more binary operations" + " and " - " on implicative algebra and obtain certain properties with these operations. Further we prove that any implicative algebra is a 
metric space. Also we prove that every implicative algebra can be made into a regular Autometrized Algebra of Swamy (1964) (see theorem 2.9).

We recall the definition of $\mathrm{Xu}$ (1993).

Defintion [2]: Let $(\mathrm{L}, \vee, \wedge, 0,1)$ be a bounded lattice with order reversing involution “' " and a binary operation $\rightarrow$ satisfying the following axioms :

$\left(\mathrm{I}_{1}\right) \mathrm{a} \rightarrow(\mathrm{b} \rightarrow \mathrm{c})=\mathrm{b} \rightarrow(\mathrm{a} \rightarrow \mathrm{c})$

(I $\mathrm{I}_{2} \mathrm{a} \rightarrow \mathrm{a}=1$

$\left(\mathrm{I}_{3}\right) \mathrm{a} \rightarrow \mathrm{b}=\mathrm{b}^{\prime} \rightarrow \mathrm{a}^{\prime}$

(I $\mathrm{I}_{4} \mathrm{a} \rightarrow \mathrm{b}=\mathrm{b} \rightarrow \mathrm{a}=1 \Rightarrow \mathrm{a}=\mathrm{b}$

$\left(\mathrm{I}_{5}\right)(\mathrm{a} \rightarrow \mathrm{b}) \rightarrow \mathrm{b} \quad=(\mathrm{b} \rightarrow \mathrm{a}) \rightarrow \mathrm{a}$

$\left(\mathrm{L}_{1}\right)(\mathrm{a} \vee \mathrm{b}) \rightarrow \mathrm{c}=(\mathrm{a} \rightarrow \mathrm{c}) \wedge(\mathrm{b} \rightarrow \mathrm{c})$

$\left(\mathrm{L}_{2}\right)(\mathrm{a} \wedge \mathrm{b}) \rightarrow \mathrm{c}=(\mathrm{a} \rightarrow \mathrm{c}) \vee(\mathrm{b} \rightarrow \mathrm{c})$.

for all a,b,c $\in \mathrm{L}$. If $(\mathrm{L}, \vee, \wedge,, 0,1)$ satisfies $\left(\mathrm{I}_{1}\right)-\left(\mathrm{I}_{5}\right)$, then $(\mathrm{L}, \vee, \wedge, 0,1)$ is said to be quasilattice implication algebra.

Now we begin with the following

Definition 1.1: An Algebra $(\mathrm{L}, \rightarrow,,, 0,1)$ of type $(2,1,0,0)$ called implicative algebra if it satisfies the following conditions

1. $\mathrm{x} \rightarrow(\mathrm{y} \rightarrow \mathrm{z})=\mathrm{y} \rightarrow(\mathrm{x} \rightarrow \mathrm{z})$

2. $1 \rightarrow \mathrm{x}=\mathrm{x}$

3. $\mathrm{x} \rightarrow 1=1$

4. $\mathrm{x} \rightarrow \mathrm{y}=\mathrm{y}^{\prime} \rightarrow \mathrm{x}^{\prime}$

5. $(\mathrm{x} \rightarrow \mathrm{y}) \rightarrow \mathrm{y}=(\mathrm{y} \rightarrow \mathrm{x}) \rightarrow \mathrm{x}$

6. $0^{\prime}=1$

for all $\mathrm{x}, \mathrm{y}, \mathrm{z} \in \mathrm{L}$

Lemma 1.2: Let $L$ be an implicative algebra. Then

1. $\mathrm{x} \rightarrow \mathrm{x}=1$ for all $\mathrm{x} \in \mathrm{L}$

2. $1^{\prime}=0$

Proof: By definition 1.1 we have $1=(\mathrm{x} \rightarrow 1) \rightarrow 1=(1 \rightarrow \mathrm{x}) \rightarrow \mathrm{x}=\mathrm{x} \rightarrow \mathrm{x}$

$$
\text { and } \begin{aligned}
1^{\prime} & =1 \rightarrow 1^{\prime} \\
& =0^{\prime} \rightarrow 1^{\prime} \\
& =1 \rightarrow 0
\end{aligned}
$$


Define a relation $\leq$ on $\mathrm{L}$ as follows $: \mathrm{x} \leq \mathrm{y} \Leftrightarrow \mathrm{x} \rightarrow \mathrm{y}=1$

Lemma 1.3: In an implicative algebra the following conditions hold

$10 \rightarrow \mathrm{x}=1$

$2 \mathrm{x} \rightarrow \mathrm{y}=1=\mathrm{y} \rightarrow \mathrm{x} \Leftrightarrow \mathrm{x}=\mathrm{y}$

$3 \mathrm{x} \rightarrow \mathrm{y}=1$ and $\mathrm{y} \rightarrow \mathrm{z}=1$ then $\mathrm{x} \rightarrow \mathrm{z}=1$

$4 \mathrm{x} \leq \mathrm{y} \Leftrightarrow \mathrm{z} \rightarrow \mathrm{x} \leq \mathrm{z} \rightarrow \mathrm{y}$ and $\mathrm{y} \rightarrow \mathrm{z} \leq \mathrm{x} \rightarrow \mathrm{z}$

$5 \quad((\mathrm{x} \rightarrow \mathrm{y}) \rightarrow \mathrm{y}) \rightarrow \mathrm{y}=\mathrm{x} \rightarrow \mathrm{y}$

6. $\quad(\mathrm{x} \rightarrow \mathrm{y}) \rightarrow[(\mathrm{y} \rightarrow \mathrm{z}) \rightarrow(\mathrm{x} \rightarrow \mathrm{z})]=1$

for all $\mathrm{x}, \mathrm{y}, \mathrm{z} \in \mathrm{L}$

Proof: We obtain all the conditions by using the definition 1.1 and lemma 1.2

1. $0 \rightarrow \mathrm{x}=\mathrm{x}^{\prime} \rightarrow 0^{\prime}$

$$
=\mathrm{x}^{\prime} \rightarrow 1=1
$$

2. Consider $\mathrm{x}=(1 \rightarrow \mathrm{x})$

$$
\begin{aligned}
& =(\mathrm{y} \rightarrow \mathrm{x}) \rightarrow \mathrm{x} \\
& =(\mathrm{x} \rightarrow \mathrm{y}) \rightarrow \mathrm{y} \\
& =1 \rightarrow \mathrm{y} \\
& =\mathrm{y}
\end{aligned}
$$

3. Let $\mathrm{x} \rightarrow \mathrm{y}=1=\mathrm{y} \rightarrow \mathrm{z}$.

Then $\mathrm{x} \rightarrow \mathrm{z}=\mathrm{x} \rightarrow(1 \rightarrow \mathrm{z})$

$$
\begin{aligned}
& =x \rightarrow((y \rightarrow z) \rightarrow z) \\
& =x \rightarrow((z \rightarrow y) \rightarrow y) \\
& =(z \rightarrow y) \rightarrow(x \rightarrow y) \\
& =(z \rightarrow y) \rightarrow 1 \\
& =1 .
\end{aligned}
$$

Hence $\mathrm{x} \leq \mathrm{z}$

4. Suppose $\mathrm{x} \leq \mathrm{y}$, then $\mathrm{x} \rightarrow \mathrm{y}=1$.

Now consider $(\mathrm{z} \rightarrow \mathrm{x}) \rightarrow(\mathrm{z} \rightarrow \mathrm{y})$

$$
\begin{aligned}
& =\left(\mathrm{x}^{\prime} \rightarrow \mathrm{z}^{\prime}\right) \rightarrow\left(\mathrm{y}^{\prime} \rightarrow \mathrm{z}^{\prime}\right) \\
& =\mathrm{y}^{\prime} \rightarrow\left(\left(\mathrm{x}^{\prime} \rightarrow \mathrm{z}^{\prime}\right) \rightarrow \mathrm{z}^{\prime}\right) \\
& =\mathrm{y}^{\prime} \rightarrow\left(\left(\mathrm{z}^{\prime} \rightarrow \mathrm{x}^{\prime}\right) \rightarrow \mathrm{x}^{\prime}\right) \\
& =\left(\mathrm{z}^{\prime} \rightarrow \mathrm{x}^{\prime}\right) \rightarrow\left(\mathrm{y}^{\prime} \rightarrow \mathrm{x}^{\prime}\right)
\end{aligned}
$$




$$
=\left(\mathrm{z}^{\prime} \rightarrow \mathrm{x}^{\prime}\right) \rightarrow 1=1
$$

Thus $\mathrm{z} \rightarrow \mathrm{x} \leq \mathrm{z} \rightarrow \mathrm{y}$

Consider $(\mathrm{y} \rightarrow \mathrm{z}) \rightarrow(\mathrm{x} \rightarrow \mathrm{z})$

$$
\begin{aligned}
& =\mathrm{x} \rightarrow((\mathrm{y} \rightarrow \mathrm{z}) \rightarrow \mathrm{z}) \\
& =\mathrm{x} \rightarrow((\mathrm{z} \rightarrow \mathrm{y}) \rightarrow \mathrm{y}) \\
& =(\mathrm{z} \rightarrow \mathrm{y}) \rightarrow(\mathrm{x} \rightarrow \mathrm{y}) \\
& =(\mathrm{z} \rightarrow \mathrm{y}) \rightarrow 1=1
\end{aligned}
$$

Hence $\mathrm{y} \rightarrow \mathrm{z} \leq \mathrm{x} \rightarrow \mathrm{z}$

From this we get that $\mathrm{x} \rightarrow \mathrm{y} \leq(\mathrm{y} \rightarrow \mathrm{z}) \rightarrow(\mathrm{x} \rightarrow \mathrm{z})$

5. $((\mathrm{x} \rightarrow \mathrm{y}) \rightarrow \mathrm{y}) \rightarrow \mathrm{y}$

$$
\begin{aligned}
& =((\mathrm{y} \rightarrow(\mathrm{x} \rightarrow \mathrm{y})) \rightarrow(\mathrm{x} \rightarrow \mathrm{y})) \\
& =((\mathrm{x} \rightarrow(\mathrm{y} \rightarrow \mathrm{y})) \rightarrow(\mathrm{x} \rightarrow \mathrm{y})) \\
& =(\mathrm{x} \rightarrow 1) \rightarrow(\mathrm{x} \rightarrow \mathrm{y})=1 \rightarrow(\mathrm{x} \rightarrow \mathrm{y})=\mathrm{x} \rightarrow \mathrm{y}
\end{aligned}
$$

6. $(\mathrm{x} \rightarrow \mathrm{y}) \rightarrow\{(\mathrm{y} \rightarrow \mathrm{z}) \rightarrow(\mathrm{x} \rightarrow \mathrm{z})\}$

$$
\begin{aligned}
& =(\mathrm{x} \rightarrow \mathrm{y}) \rightarrow\{\mathrm{x} \rightarrow\{(\mathrm{y} \rightarrow \mathrm{z}) \rightarrow \mathrm{z})\}\} \\
& =(\mathrm{x} \rightarrow \mathrm{y}) \rightarrow\{\mathrm{x} \rightarrow\{(\mathrm{z} \rightarrow \mathrm{y}) \rightarrow \mathrm{y})\}\} \\
& =(\mathrm{x} \rightarrow \mathrm{y}) \rightarrow\{(\mathrm{z} \rightarrow \mathrm{y}) \rightarrow(\mathrm{x} \rightarrow \mathrm{y})\} \\
& =(\mathrm{z} \rightarrow \mathrm{y}) \rightarrow\{(\mathrm{x} \rightarrow \mathrm{y}) \rightarrow(\mathrm{x} \rightarrow \mathrm{y})\}=(\mathrm{z} \rightarrow \mathrm{y}) \rightarrow 1=1 .
\end{aligned}
$$

Remark 1.4 : From condition 1 of lemma 1.2 , conditions 2 and 3 of lemma 1.3 , it is clear that $\mathrm{L}$ is a partially ordered set and from conditions 3 of definition 1.1 and condition 1 of lemma 1.3 ,L is bounded. Thus $\mathrm{L}$ is a bounded poset.

Lemma 1.5: Let $\mathrm{L}$ be implicative algebra, then $\left(\mathrm{x}^{\prime}\right)^{\prime}=\mathrm{x}$ for all $\mathrm{x} \in \mathrm{L}$

Proof: Using the conditions 2,4 and 6 of definition 1.1, and 2 of lemma 1.2 we have

$$
\begin{aligned}
\left(\mathrm{x}^{\prime}\right)^{\prime} & =1 \rightarrow\left(\mathrm{x}^{\prime}\right)^{\prime} \\
& =0^{\prime} \rightarrow\left(\mathrm{x}^{\prime}\right)^{\prime} \\
& =\mathrm{x}^{\prime} \rightarrow 0 \\
& =\mathrm{x}^{\prime} \rightarrow 1^{\prime} \\
& =1 \rightarrow \mathrm{x} \\
& =\mathrm{x}
\end{aligned}
$$

Corollary 1.6: In any implicative algebra, $x^{\prime}=x \rightarrow 0$ for all $x$ in $L$ 
Proof: $\mathrm{x}^{\prime}=1 \rightarrow \mathrm{x}^{\prime}=\left(\mathrm{x}^{\prime}\right)^{\prime} \rightarrow 1^{\prime}=\mathrm{x} \rightarrow 0$

Now we define two binary operations $\vee$ and $\wedge$ on an implicative algebra L by

$\mathrm{x} \vee \mathrm{y}=(\mathrm{x} \rightarrow \mathrm{y}) \rightarrow \mathrm{y}=(\mathrm{y} \rightarrow \mathrm{x}) \rightarrow \mathrm{x}$

$\mathrm{x} \wedge \mathrm{y}=\left((\mathrm{y} \rightarrow \mathrm{x}) \rightarrow \mathrm{y}^{\prime}\right)^{\prime}=\left((\mathrm{x} \rightarrow \mathrm{y}) \rightarrow \mathrm{x}^{\prime}\right)^{\prime}$

Theorem 1.7: In any implicative algebra $\mathrm{L}$, the following hold for all $\mathrm{x}, \mathrm{y} \in \mathrm{L}$

1. $(\mathrm{x} \vee \mathrm{y})^{\prime}=\mathrm{x}^{\prime} \wedge \mathrm{y}^{\prime}$

2. $(x \wedge y)^{\prime}=x^{\prime} \vee y^{\prime} \quad$ for all $x, y \in L$

\section{Proof of}

1. Consider $(\mathrm{x} \vee \mathrm{y})^{\prime} \rightarrow\left(\mathrm{x}^{\prime} \wedge \mathrm{y}^{\prime}\right)$

$$
\begin{aligned}
& =((x \rightarrow y) \rightarrow y)^{\prime} \rightarrow\left(\left(y^{\prime} \rightarrow x^{\prime}\right) \rightarrow y^{\prime}\right)^{\prime} \\
& =\left(\left(y^{\prime} \rightarrow x^{\prime}\right) \rightarrow y\right) \rightarrow((x \rightarrow y) \rightarrow y) \\
& =((x \rightarrow y) \rightarrow y) \rightarrow((x \rightarrow y) \rightarrow y)=1 \\
& \text { And }\left(x^{\prime} \wedge y^{\prime}\right) \rightarrow(x \vee y)^{\prime} \\
& =\left(\left(y^{\prime} \rightarrow x^{\prime}\right) \rightarrow y^{\prime \prime}\right)^{\prime} \rightarrow((x \rightarrow y) \rightarrow y)^{\prime} \\
& =((x \rightarrow y) \rightarrow y) \rightarrow((x \rightarrow y) \rightarrow y)=1
\end{aligned}
$$

2. Consider $(\mathrm{x} \wedge \mathrm{y})^{\prime} \rightarrow\left(\mathrm{x}^{\prime} \vee \mathrm{y}^{\prime}\right)=\left\{\left((\mathrm{y} \rightarrow \mathrm{x}) \rightarrow \mathrm{y}^{\prime}\right)^{\prime}\right\}^{\prime} \rightarrow\left(\left(\mathrm{x}^{\prime} \rightarrow \mathrm{y}^{\prime}\right) \rightarrow \mathrm{y}^{\prime}\right)$

$$
\begin{aligned}
& =\left((y \rightarrow x) \rightarrow y^{\prime}\right) \rightarrow\left(\left(x^{\prime} \rightarrow y^{\prime}\right) \rightarrow y^{\prime}\right) \\
& =\left((y \rightarrow x) \rightarrow y^{\prime}\right) \rightarrow\left((y \rightarrow x) \rightarrow y^{\prime}\right)=1 \\
\text { and }\left(x^{\prime} \vee y^{\prime}\right) \rightarrow(x \wedge y)^{\prime} & =\left(\left(x^{\prime} \rightarrow y^{\prime}\right) \rightarrow y^{\prime}\right) \rightarrow\left\{\left((y \rightarrow x) \rightarrow y^{\prime}\right)^{\prime}\right\}^{\prime} \\
& =\left(\left(x^{\prime} \rightarrow y^{\prime}\right) \rightarrow y^{\prime}\right) \rightarrow\left((y \rightarrow x) \rightarrow y^{\prime}\right) \\
& =\left(\left(x^{\prime} \rightarrow y^{\prime}\right) \rightarrow y^{\prime}\right) \rightarrow\left(\left(x^{\prime} \rightarrow y^{\prime}\right) \rightarrow y^{\prime}\right)=1
\end{aligned}
$$

Hence by 2 of lemma 1.3 the result follows

Lemma 1.8: In any implicative algebra L, the following hold

1. $\mathrm{x} \wedge \mathrm{y} \leq \mathrm{x}, \mathrm{y} \leq \mathrm{x} \vee \mathrm{y}$

2. $\mathrm{x} \vee \mathrm{y}$ is the least upper bound of $\{\mathrm{x}, \mathrm{y}\}$

3. $\mathrm{x} \wedge \mathrm{y}$ is the greatest lower bound for $\{\mathrm{x}, \mathrm{y}\}$

for all $\mathrm{x}, \mathrm{y} \in \mathrm{L}$

\section{Proof:}

1. $(\mathrm{x} \wedge \mathrm{y}) \rightarrow \mathrm{x}=\left((\mathrm{y} \rightarrow \mathrm{x}) \rightarrow \mathrm{y}^{\prime}\right)^{\prime} \rightarrow \mathrm{x}$

$$
=x^{\prime} \rightarrow\left((y \rightarrow x) \rightarrow y^{\prime}\right)
$$




$$
\begin{aligned}
& \qquad(y \rightarrow x) \rightarrow\left(x^{\prime} \rightarrow y^{\prime}\right) \\
& =(y \rightarrow x) \rightarrow(y \rightarrow x)=1 \quad \text { and } \\
& \begin{aligned}
(x \wedge y) \rightarrow y & =\left((y \rightarrow x) \rightarrow y^{\prime}\right)^{\prime} \rightarrow y \\
= & y^{\prime} \rightarrow\left((y \rightarrow x) \rightarrow y^{\prime}\right) \\
= & (y \rightarrow x) \rightarrow\left(y^{\prime} \rightarrow y^{\prime}\right) \\
= & (y \rightarrow x) \rightarrow 1=1
\end{aligned} \\
& \text { Further } x \rightarrow x \vee y=x \rightarrow((x \rightarrow y) \rightarrow y) \\
& \qquad=(x \rightarrow y) \rightarrow(x \rightarrow y)=1 \text { and } \\
& y \rightarrow x \vee y=y \rightarrow((x \rightarrow y) \rightarrow y)=(x \rightarrow y) \rightarrow(y \rightarrow y)=(x \rightarrow y) \rightarrow 1=1
\end{aligned}
$$

2. From 1, it can be observed that $\mathrm{x} \vee \mathrm{y}$ is an upper bound for $\{\mathrm{x}, \mathrm{y}\}$.

Let $\mathrm{t}$ be any upper bound for $\mathrm{x}, \mathrm{y}$ then $\mathrm{x}, \mathrm{y} \leq \mathrm{t}$. This implies $\mathrm{x} \rightarrow \mathrm{t}=1=\mathrm{y} \rightarrow \mathrm{t}$.

Using definition 1.1 , conditions 5 and 6 of lemma 1.3 and (b) of remark 1.4 , we get

$$
\begin{aligned}
(\mathrm{x} \vee \mathrm{y}) \rightarrow \mathrm{t}= & ((\mathrm{x} \rightarrow \mathrm{y}) \rightarrow \mathrm{y}) \rightarrow \mathrm{t} \\
& =(\mathrm{x} \rightarrow \mathrm{y}) \rightarrow \mathrm{y}) \rightarrow(1 \rightarrow \mathrm{t}) \\
& =((\mathrm{x} \rightarrow \mathrm{y}) \rightarrow \mathrm{y}) \rightarrow((\mathrm{y} \rightarrow \mathrm{t}) \rightarrow \mathrm{t}) \\
& =((\mathrm{x} \rightarrow \mathrm{y}) \rightarrow \mathrm{y}) \rightarrow((\mathrm{t} \rightarrow \mathrm{y}) \rightarrow \mathrm{y}) \\
& =(\mathrm{t} \rightarrow \mathrm{y}) \rightarrow((\mathrm{x} \rightarrow \mathrm{y}) \rightarrow \mathrm{y}) \rightarrow \mathrm{y}) \\
& =(\mathrm{t} \rightarrow \mathrm{y}) \rightarrow(\mathrm{x} \rightarrow \mathrm{y}) \\
& \geq \mathrm{x} \rightarrow \mathrm{t}=1
\end{aligned}
$$

Hence $(x \vee y) \rightarrow t=1$

Thus $\mathrm{x} \vee \mathrm{yt} \leq \mathrm{t}$. This shows that $\mathrm{x} \vee \mathrm{y}$ is the least upper bound of $\{\mathrm{x}, \mathrm{y}\}$

3. Clearly . $\mathrm{x} \wedge \mathrm{y}$ is a lower bound for $\{\mathrm{x}, \mathrm{y}\}$.Suppose $\mathrm{t}$ is any lower bound for $\{\mathrm{x}, \mathrm{y}\}$

Then $\mathrm{t} \leq \mathrm{x}$ and $\mathrm{t} \leq \mathrm{y}$. This means $\mathrm{t} \rightarrow \mathrm{x}=1=\mathrm{t} \rightarrow \mathrm{y}$.

Consider $\mathrm{t} \rightarrow \mathrm{x} \wedge \mathrm{y}$

$$
\begin{aligned}
& =\mathrm{t} \rightarrow\left((\mathrm{y} \rightarrow \mathrm{x}) \rightarrow \mathrm{y}^{\prime}\right)^{\prime} \\
& =\left((\mathrm{y} \rightarrow \mathrm{x}) \rightarrow \mathrm{y}^{\prime}\right) \rightarrow \mathrm{t}^{\prime} \quad \text { ( by } 4 \text { of definition 1.1) } \\
& =\left((\mathrm{y} \rightarrow \mathrm{x}) \rightarrow \mathrm{y}^{\prime}\right) \rightarrow\left(1 \rightarrow \mathrm{t}^{\prime}\right) \quad \text { ( by } 2 \text { of definition 1.1) } \\
& =\left((\mathrm{y} \rightarrow \mathrm{x}) \rightarrow \mathrm{y}^{\prime}\right) \rightarrow\left(\left(\mathrm{y}^{\prime} \rightarrow \mathrm{t}^{\prime}\right) \rightarrow \mathrm{t}^{\prime}\right) \quad(\text { by assumption }) \\
& =\left((\mathrm{y} \rightarrow \mathrm{x}) \rightarrow \mathrm{y}^{\prime}\right) \rightarrow\left(\left(\mathrm{t}^{\prime} \rightarrow \mathrm{y}^{\prime}\right) \rightarrow \mathrm{y}^{\prime}\right) \quad(\text { by } 5 \text { of definition } 1.1) \\
& \geq\left(\mathrm{t}^{\prime} \rightarrow \mathrm{y}^{\prime}\right) \rightarrow((\mathrm{y} \rightarrow \mathrm{x}) \quad \text { ( by } 6 \text { of lemma } 1.3) \\
& =\left(\mathrm{t}^{\prime} \rightarrow \mathrm{y}^{\prime}\right) \rightarrow\left(\left(\mathrm{x}^{\prime} \rightarrow \mathrm{y}^{\prime}\right) \quad \text { ( by } 4 \text { of definition } 1.1\right)
\end{aligned}
$$




$$
\geq\left(\mathrm{x}^{\prime} \rightarrow \mathrm{t}^{\prime}\right)=1
$$

Thus $\mathrm{t} \leq \mathrm{x} \wedge \mathrm{y}$. Hence the result

Remark 1.9: The condition 2 of lemma 1.8 guarantees that every two elements in an implicative algebra has supremum and infimum. Hence $(\mathrm{L}, \leq)$ is a bounded lattice with bounds 0 and 1 .

Now we have the following corollaries 1.10 and 1.11 as a consequence of lemma 1.8

\section{Corollary1.10:}

1. $\mathrm{x} \leq \mathrm{y}, \mathrm{x} \leq \mathrm{z} \Rightarrow \mathrm{x} \leq \mathrm{y} \wedge \mathrm{z}$

2. $\mathrm{y} \leq \mathrm{x}, \mathrm{z} \leq \mathrm{x} \Rightarrow \mathrm{y} \vee \mathrm{z} \leq \mathrm{x}$

Corollary 1.11: In any implicative algebra $L$, the following hold (for all $x, y, z \in L$ )

1. $(\mathrm{x} \vee \mathrm{y}) \rightarrow \mathrm{z} \leq \mathrm{x} \rightarrow \mathrm{z}$ and $(\mathrm{x} \vee \mathrm{y}) \rightarrow \mathrm{z} \leq \mathrm{y} \rightarrow \mathrm{z}$

2. $\mathrm{x} \rightarrow \mathrm{z} \leq(\mathrm{x} \wedge \mathrm{y}) \rightarrow \mathrm{z}$ and $\mathrm{y} \rightarrow \mathrm{z} \leq(\mathrm{x} \wedge \mathrm{y}) \rightarrow \mathrm{z}$

Theorem 1.12: In an implicative algebra $\mathrm{L}$, the following conditions hold: (for all $\mathrm{x}, \mathrm{y}, \mathrm{z} \in \mathrm{L}$ )

1. $(\mathrm{x} \vee \mathrm{y}) \rightarrow \mathrm{z}=(\mathrm{x} \rightarrow \mathrm{z}) \wedge(\mathrm{y} \rightarrow \mathrm{z})$

2. $(\mathrm{x} \wedge \mathrm{y}) \rightarrow \mathrm{z}=(\mathrm{x} \rightarrow \mathrm{z}) \vee(\mathrm{y} \rightarrow \mathrm{z})$

Proof: From the above corollary 1.10 and 4 of lemma 1.3 it is clear that

$$
\begin{aligned}
& (\mathrm{x} \vee \mathrm{y}) \rightarrow \mathrm{z} \leq(\mathrm{x} \rightarrow \mathrm{z}) \wedge(\mathrm{y} \rightarrow \mathrm{z}) \\
& \text { To prove the other way round, consider the following } \\
& \{(\mathrm{x} \rightarrow \mathrm{z}) \wedge(\mathrm{y} \rightarrow \mathrm{z})\} \rightarrow\{(\mathrm{x} \vee \mathrm{y}) \rightarrow \mathrm{z}) \\
& \left.\left.=[(\mathrm{x} \rightarrow \mathrm{z}) \rightarrow(\mathrm{y} \rightarrow \mathrm{z})) \rightarrow(\mathrm{x} \rightarrow \mathrm{z})^{\prime}\right]^{\prime} \rightarrow((\mathrm{x} \rightarrow \mathrm{y}) \rightarrow \mathrm{y}) \rightarrow \mathrm{z}\right) \\
& =((\mathrm{x} \rightarrow \mathrm{y}) \rightarrow \mathrm{y}) \rightarrow \mathrm{z})^{\prime} \rightarrow[(\mathrm{x} \rightarrow \mathrm{z}) \rightarrow(\mathrm{y} \rightarrow \mathrm{z})) \rightarrow\left((\mathrm{x} \rightarrow \mathrm{z})^{\prime}\right] \text { (by } 4 \text { of def } 1.1 \& \text { lemma 1.5) } \\
& \left.\left.=\{(\mathrm{x} \rightarrow \mathrm{z}) \rightarrow(\mathrm{y} \rightarrow \mathrm{z})\} \rightarrow[(\mathrm{x} \rightarrow \mathrm{y}) \rightarrow \mathrm{y}) \rightarrow \mathrm{z})^{\prime} \rightarrow(\mathrm{x} \rightarrow \mathrm{z})^{\prime}\right\} \text { (by } 1 \text { of } \operatorname{def} 1.1\right) \\
& =\{(\mathrm{x} \rightarrow \mathrm{z}) \rightarrow(\mathrm{y} \rightarrow \mathrm{z})\} \rightarrow\{[(\mathrm{x} \rightarrow \mathrm{z}) \rightarrow((\mathrm{x} \rightarrow \mathrm{y}) \rightarrow \mathrm{y}) \rightarrow \mathrm{z})\} \quad \text { (by } 4 \text { of def } 1.1) \\
& =((\mathrm{y} \rightarrow((\mathrm{x} \rightarrow \mathrm{z}) \rightarrow \mathrm{z})) \rightarrow[(\mathrm{x} \rightarrow \mathrm{y}) \rightarrow \mathrm{y}) \rightarrow((\mathrm{x} \rightarrow \mathrm{z}) \rightarrow \mathrm{z})] \quad \text { (by } 1 \text { of def } 1.1) \\
& =((\mathrm{x} \rightarrow \mathrm{y}) \rightarrow \mathrm{y}) \rightarrow\{[\mathrm{y} \rightarrow((\mathrm{x} \rightarrow \mathrm{z}) \rightarrow \mathrm{z})] \rightarrow((\mathrm{x} \rightarrow \mathrm{z}) \rightarrow \mathrm{z})] \quad \text { (by } 1 \text { of def 1.1) } \\
& =((\mathrm{x} \rightarrow \mathrm{y}) \rightarrow \mathrm{y}) \rightarrow\{[((\mathrm{x} \rightarrow \mathrm{z}) \rightarrow \mathrm{z})] \rightarrow \mathrm{y}) \rightarrow \mathrm{y}] \quad \text { (by } 5 \text { of def } 1.1) \\
& =((\mathrm{x} \rightarrow \mathrm{z}) \rightarrow \mathrm{z}) \rightarrow \mathrm{y}) \rightarrow((\mathrm{x} \rightarrow \mathrm{y}) \rightarrow \mathrm{y}) \rightarrow \mathrm{y}) \quad \text { (by } 1 \text { of def } 1.1) \\
& =((\mathrm{x} \rightarrow \mathrm{z}) \rightarrow \mathrm{z}) \rightarrow \mathrm{y}) \rightarrow(\mathrm{x} \rightarrow \mathrm{y}) \quad \text { (by } 5 \text { of lemma 1.3) } \\
& \geq \mathrm{x} \rightarrow((\mathrm{x} \rightarrow \mathrm{z}) \rightarrow \mathrm{z}) \\
& \text { (by } 6 \text { of lemma 1.3) } \\
& =(\mathrm{x} \rightarrow \mathrm{z}) \rightarrow(\mathrm{x} \rightarrow \mathrm{z}) \\
& \text { (by } 1 \text { of def 1.1) } \\
& =1
\end{aligned}
$$

Hence $(x \rightarrow z) \wedge(y \rightarrow z) \leq(x \vee y) \rightarrow z$ 
This completes the proof.

(2) Follows in the similar lines of (1)

From remark 1.9 and theorem 1.11, we have the following

Theorem 1.13: Let $\left(\mathrm{L}, \rightarrow,,^{\prime}, 0,1\right)$ be an implicative algebra . Then $(\mathrm{L}, \vee, \wedge, 0,1)$ is a lattice implication algebra.

Remark 1.14: Let $\left(\mathrm{L}, \rightarrow,,^{\prime}, 0,1\right)$ be an implicative algebra with $0 \neq 1$ then $\rightarrow$ can never be associative.

Suppose $\rightarrow$ is associative. Let $\mathrm{a}=\mathrm{b}=\mathrm{c}$ in 1 of definition 1.1

then $(\mathrm{a} \rightarrow \mathrm{a}) \rightarrow \mathrm{a}=\mathrm{a} \rightarrow(\mathrm{a} \rightarrow \mathrm{a})$

$\Rightarrow \quad 1 \rightarrow \mathrm{a}=\mathrm{a} \rightarrow 1$

$\Rightarrow \quad \mathrm{a}=1$. Hence $0=1$, a contradiction

\section{AUTOMETRIZATION ON IMPLICATIVE ALGEBRAS}

In this article we introduce two binary operations on implicative algebras namely + and - we obtain a few results concerning the operations defined. Also we obtain some geometric properties of implicative algebra.

We begin with the following.

Let $\mathrm{L}$ be implicative algebra. Define + and - on $\mathrm{L}$ as follows .

For $\mathrm{x}, \mathrm{y}$ in $\mathrm{L}, \mathrm{x}-\mathrm{y}=(\mathrm{x} \rightarrow \mathrm{y})^{\prime} \quad$ and

$$
\mathrm{x}+\mathrm{y}=\mathrm{x}^{\prime} \rightarrow \mathrm{y}=\mathrm{y}^{\prime} \rightarrow \mathrm{x}
$$

Observe that $\mathrm{x}+\mathrm{y}=\mathrm{y}+\mathrm{x}$

Then we obtain the following in

Lemma 2.1: Let $\mathrm{L}$ be implicative algebra .Then $(\mathrm{L},+, 0)$ is a commutative monoid

Proof: Let $\mathrm{x}, \mathrm{y}, \mathrm{z} \in \mathrm{L}$. Then $(\mathrm{x}+\mathrm{y})+\mathrm{z}=\left\{\left(\mathrm{x}^{\prime} \rightarrow \mathrm{y}\right)^{\prime} \rightarrow \mathrm{z}\right\}$

$$
\begin{aligned}
& =\mathrm{z}^{\prime} \rightarrow\left(\mathrm{x}^{\prime} \rightarrow \mathrm{y}\right) \\
& =\mathrm{x}^{\prime} \rightarrow\left(\mathrm{z}^{\prime} \rightarrow \mathrm{y}\right) \\
& =\mathrm{x}^{\prime} \rightarrow\left(\mathrm{y}^{\prime} \rightarrow \mathrm{z}\right) \\
& =\mathrm{x}+(\mathrm{y}+\mathrm{z}) .
\end{aligned}
$$

Also $\mathrm{x}+0=\mathrm{x}^{\prime} \rightarrow 0=0^{\prime} \rightarrow \mathrm{x}=1 \rightarrow \mathrm{x}=\mathrm{x}$.

Also we have the easy consequences of the definition of - and with + in the following

Lemma 2.2: For a,b,c, $x$ and $y$ in an implicative algebra L, the following conditions hold. 
1. $\mathrm{a}-\mathrm{a}=0$

2. $a-0=a$

3. $(\mathrm{a}-\mathrm{b}) \vee 0=\mathrm{a}-\mathrm{b}$

4. $a-b \leq 0 \Leftrightarrow a \leq b$

5. $(\mathrm{a} \vee \mathrm{b})-\mathrm{c}=(\mathrm{a}-\mathrm{c}) \vee(\mathrm{b}-\mathrm{c})$

6. $a \vee b=(a-b)+b$

7. $a \wedge b=b-(b-a)$

8. $x \leq a+b \Leftrightarrow x-a \leq b$

9. $0-\mathrm{a}=0$

10. $a-(a+b)=(a-b)-c$

11. $(a-b)+(b-c) \geq a-c$

12. $\mathrm{a}=\mathrm{a} \vee 0+\mathrm{a} \wedge 0$

13. $a-(b+c)=(a-c)-b$

14. $a \geq b \Rightarrow(a-b)+b=a$

15. $(a-(x \wedge y))+b=((a-x)+b) \vee((a-y)+b)$

Now we are in a position to introduce the concept of a metric on an implicative algebra

Definiton 2.3: Let $\mathrm{L}$ be an implicative algebra. Define a map $\quad *: \mathrm{L} x \mathrm{~L} \rightarrow \mathrm{L}$ by $a * b=(a-b)+(b-a)$

Lemma 2.4: In an implicative algebra $\mathrm{L},(\mathrm{a} \rightarrow \mathrm{b}) \rightarrow(\mathrm{b} \rightarrow \mathrm{a})^{\prime}=0 \Rightarrow \mathrm{a}=\mathrm{b}$

Proof: Consider $\mathrm{a} \rightarrow \mathrm{b}=1 \rightarrow(\mathrm{a} \rightarrow \mathrm{b})$

$$
\begin{aligned}
& =(a \rightarrow b)^{\prime} \rightarrow 1^{\prime} \\
& =(a \rightarrow b)^{\prime} \rightarrow 0 \\
& =(a \rightarrow b)^{\prime} \rightarrow\left\{(a \rightarrow b) \rightarrow(b \rightarrow a)^{\prime}\right\} \\
& =(a \rightarrow b) \rightarrow\left\{(a \rightarrow b)^{\prime} \rightarrow(b \rightarrow a)^{\prime}\right\} \\
& =(a \rightarrow b) \rightarrow\{(b \rightarrow a) \rightarrow(a \rightarrow b)\} \\
& =(b \rightarrow a) \rightarrow\{(a \rightarrow b) \rightarrow(a \rightarrow b)\} \\
& =(b \rightarrow a) \rightarrow 1=1 \text {. Hence } a \leq b . \text { Similarly we obtain } b \leq a \text {. Thus } a=b
\end{aligned}
$$

Theorem.2.5: Let $\mathrm{L}$ be an implicative algebra . Then for all a,b,c in $\mathrm{L}$

$$
\mathrm{a} \leq \mathrm{b} \Rightarrow \mathrm{a}+\mathrm{c} \leq \mathrm{b}+\mathrm{c}
$$

Proof: Let $\mathrm{a} \leq \mathrm{b} \Rightarrow \mathrm{a} \rightarrow \mathrm{b}=1$.

Consider $\{(\mathrm{a}+\mathrm{b}) \rightarrow(\mathrm{b}+\mathrm{c})\}=\left(\mathrm{a}^{\prime} \rightarrow \mathrm{c}\right) \rightarrow\left(\mathrm{b}^{\prime} \rightarrow \mathrm{c}\right)=\mathrm{b}^{\prime} \rightarrow\left(\left(\mathrm{a}^{\prime} \rightarrow \mathrm{c}\right) \rightarrow \mathrm{c}\right)$ 


$$
=\mathrm{b}^{\prime} \rightarrow\left(\left(\mathrm{c} \rightarrow \mathrm{a}^{\prime}\right) \rightarrow \mathrm{a}^{\prime}\right)=\left(\mathrm{c} \rightarrow \mathrm{a}^{\prime}\right) \rightarrow\left(\mathrm{b}^{\prime} \rightarrow \mathrm{a}^{\prime}\right)=\left(\mathrm{c} \rightarrow \mathrm{a}^{\prime}\right) \rightarrow 1=1 \text {. Hence } \mathrm{a}+\mathrm{c} \leq \mathrm{b}+\mathrm{c}
$$

A simple consequence of the above theorem 2.4 is given as

Corallary 2.6: In any implicative algebra L,

$$
\mathrm{a} \leq \mathrm{b} \text { and } \mathrm{c} \leq \mathrm{d} \Rightarrow \mathrm{a}+\mathrm{c} \leq \mathrm{b}+\mathrm{d} \text { for all } \mathrm{a}, \mathrm{b}, \mathrm{c}, \mathrm{d} \in \mathrm{L}
$$

Now we have the following

Theorem 2.7: $(\mathrm{L}, *)$ is a metric space

Proof: Let $\mathrm{a}, \mathrm{b}, \mathrm{c} \in \mathrm{L}$. Clearly $(\mathrm{a} \rightarrow \mathrm{b})^{\prime} \geq 0$. Thus

$a * b=(a-b)+(b-a)=(a \rightarrow b)^{\prime}+(a \rightarrow b)^{\prime} \geq 0$ and also $a * b=b * a$

Let $a * b=0$. Then from lemma 2.3 , it follows that $a=b$

Conversely suppose $\mathrm{a}=\mathrm{b}$ then $\mathrm{a} * \mathrm{a}=0$ since $\mathrm{a}-\mathrm{a}=0$

$$
\text { Finally, } \begin{aligned}
(\mathrm{a} * \mathrm{~b})=(\mathrm{b} * \mathrm{a}) & =\{(\mathrm{a}-\mathrm{b})+(\mathrm{b}-\mathrm{a})\}+\{(\mathrm{b}-\mathrm{a})+(\mathrm{c}-\mathrm{b}\} \\
& =\{(\mathrm{a}-\mathrm{b})+(\mathrm{b}-\mathrm{c})\}+\{(\mathrm{c}-\mathrm{b})+(\mathrm{b}-\mathrm{a}\} \\
& \geq(\mathrm{a}-\mathrm{c})+(\mathrm{c}-\mathrm{a})(\text { by corollary } 2.6) \\
& =\mathrm{a} * \mathrm{c} . \text { Thus } * \text { is a metric on } \mathrm{L}
\end{aligned}
$$

We recall the following from Swamy (1964).

Definition [1]: A system $\mathrm{A}=(\mathrm{A},+, \leq, *)$ is called an "Autometrized Algebra" if and only if $1.1(\mathrm{~A},+)$ is a binary commutative algebra with a distinguished element Zero :" 0 “

$1.2 \leq$ is an anti symmetric, reflexive ordering on $\mathrm{A}$ and

$1.3 *$ : A X A is a mapping satisfying the formal properties of a distance function namely:

1. $\mathrm{a} * \mathrm{~b} \geq 0$ with equality $\Leftrightarrow a=b \quad 2 . \mathrm{a} * \mathrm{~b}=\mathrm{b} * \mathrm{a}$ and $3 \mathrm{a} * \mathrm{c} \leq \mathrm{a} * \mathrm{~b}+\mathrm{b} * \mathrm{c}$

Definition [1]: An Autometrized Algebra is said to be regular if $\mathrm{a} * 0=\mathrm{a}$

Theorem 2.8: Let $\mathrm{L}$ be an implicative algebra. Then $\mathrm{a} * 0=\mathrm{a}$ for all $\mathrm{a} \in \mathrm{L}$

Proof: $a * 0=(a-0)+(0-a)=a+0($ by lemma 2.2$)=a$.

Thus from remark 1.4, Lemma 2.1, theorem 2.6, and theorem 2.7 , we have the following

Theorem 2.9: Any implicative algebra L is a regular Autometrized Algebra

We end this section by looking at the following

Example 2.10 : Let $\mathrm{L}=\{0, \mathrm{a}, \mathrm{b}, \mathrm{c}, 1\}$ be a chain defined by $0<\mathrm{a}<\mathrm{b}<\mathrm{c}<1$

Define ' and $\rightarrow$ and $*$ as follows 


\begin{tabular}{|l|l|}
\hline l & 0 \\
\hline 0 & 1 \\
\hline a & c \\
\hline b & b \\
\hline c & a \\
\hline 1 & 0 \\
\hline
\end{tabular}

\begin{tabular}{|l|l|l|l|l|l|}
\hline$\rightarrow$ & 0 & $\mathrm{a}$ & $\mathrm{b}$ & $\mathrm{c}$ & 1 \\
\hline 0 & 1 & 1 & 1 & 1 & 1 \\
\hline $\mathrm{a}$ & $\mathrm{c}$ & 1 & 1 & 1 & 1 \\
\hline $\mathrm{b}$ & $\mathrm{b}$ & $\mathrm{c}$ & 1 & 1 & 1 \\
\hline $\mathrm{c}$ & $\mathrm{a}$ & $\mathrm{b}$ & $\mathrm{c}$ & 1 & 1 \\
\hline 1 & 0 & $\mathrm{a}$ & $\mathrm{b}$ & $\mathrm{c}$ & 1 \\
\hline
\end{tabular}

\begin{tabular}{|l|l|l|l|l|l|}
\hline$*$ & 0 & a & b & c & 1 \\
\hline 0 & 0 & a & b & c & 1 \\
\hline a & 0 & 0 & a & b & c \\
\hline b & b & a & 0 & a & b \\
\hline c & c & b & a & 0 & a \\
\hline 1 & 1 & c & b & a & 0 \\
\hline
\end{tabular}

Clearly 0 is the additive element as $\mathrm{x} * 0=0 * \mathrm{x}=\mathrm{x}$ for all $\mathrm{x}$ and every element is the inverse of itself since $\mathrm{x} * \mathrm{x}=0$ for all $\mathrm{x}$. Further it is observed that $*$ is not associative. For instance $(\mathrm{a} * \mathrm{~b}) * \mathrm{c}=\mathrm{b} \neq 0=\mathrm{a} *(\mathrm{~b} * \mathrm{c})$.

Thus $*$ is not associative. Hence $*$ is not a group operation.

\section{CONCLUSION}

We obtained the equivalent definition of lattice implication algebra of $\mathrm{Xu}$ (1993) with fewer axioms and proved that every lattice implication algebra is an Autometrized Algebra of Swamy (1964).

\section{ACKNOWLEDGEMENTS}

We sincerely thank the referees for their valuable suggestions and critical comments regarding this paper.

\section{REFERENCES}


Jun,Y.B., Xu, Yang \& Keyun Qin. 1998. Positive implication and Associative filters of lattice implication algebras. Bull. Korean Math. Soc., 35(1): 53-61.

Liu, J \& Xu, Y. 1997. Filters and structure of lattice implication algebra. Chinese Science Bull., 42:1517-1520.

Swamy, K. L. N. 1964. A General theory of Autometrized Algebras. Math Annallen, 157: 6574.

Xu, Pan. Y. 2008. Lattice implication ordered semigroups. Information Sci., 178:403-413.

Xu, Y. 1993. Lattice implication algebras. J South West Jiaotong University, 28(1): 20- 27.

Zhu, H., Zhao, J.B \& Xu, Y. 2006. The primary ideal of residuated lattice implication algebra. Journal of Zhengzhou University, 38(2): 25-28.

Zhu Yiquan \& Tu Wenbiao. 2001. A Note on lattice implication algebras. Bull. Korean Math. Soc., 38 (1):191-195 